\title{
Malignant priapism due to penile metastases: Case series and literature review
}

\author{
Francesco De Luca ${ }^{1}$, Evangelos Zacharakis ${ }^{2}$, Majed Shabbir ${ }^{2}$, Angela Maurizi ${ }^{3}$, Emy Manzi ${ }^{4}$, \\ Antonio Zanghì ${ }^{5}$, Carlo De Dominicis ${ }^{3}$, David Ralph ${ }^{1}$ \\ ${ }^{1}$ St Peter's Andrology and the Insitute of Urology, University College London Hospital, London, UK; \\ 2 Department of Urology, Guy's Hospital, Kings College London, UK; \\ ${ }^{3}$ Department of Gynecologic-Obstetric Sciences and Urological Sciences, Sapienza University of Rome, Rome, Italy; \\ ${ }^{4}$ Department of General Surgery and Organ Transplantation Sapienza University of Rome, Rome, Italy; \\ ${ }^{5}$ General Surgery and Breast Unit, Department of Surgery University of Catania, Catania, Italy.
}

\begin{abstract}
Summary Malignant priapism secondary to penile metastases is a rare condition. This term was originally used by Peacock in 1938 to describe a condition of painful induration and erection of the penis due to metastatic infiltration by a neoplasm. In the current literature there are 512 case reports. The primary tumor sites are bladder, prostate and rectum. The treatment has only palliative intent and consists of local tumor excision, penectomy, radiotherapy and chemotherapy. We present one case of malignant priapism originated from prostate cancer, and two from urothelial carcinoma of the bladder. Different approaches in diagnosis and therapy were performed. The entire three patient reported a relief of the pain following the treatment, with an improvement of their quality of life, even though it was only temporary as a palliative. Malignant priapism is a rare medical emergency. Penile/pelvis magnetic resonance imaging (MRI) scan and corporal biopsies are considered an effective method of diagnosis of the primary organ site.
\end{abstract}

KEY WORDS: Priapism; Tumour; Metastases.

Submitted 19 October 2015; Accepted 8 December 2015

\section{INTRODUCTION}

Malignant priapism secondary to penile metastases is a rare condition. This term was originally used by Peacock in 1938 to describe a condition of painful induration and erection of the penis due to infiltration by metastatic neoplasm. Clinical manifestations of penile metastasis are priapism, painful or painless palpable penile nodules, swelling, haematuria and urinary retention. In the current literature there are 512 case reports. The primary tumor sites are usually the bladder, prostate and rectum. The treatment has only palliative intent and consists of hormone therapy, chemotherapy, radiotherapy, local tumor excision and penectomy.

\section{Presentation of case(s)}

We reported three cases of malignant priapism.

Case 1: a 62 year old man with diabetes mellitus type II and a history of prostate cancer underwent laparoscopic radical prostatectomy, followed by salvage radiotherapy. The histopathology report showed advanced disease with Gleason score $9(4+5)$. As a result of his prostatectomy and radiation therapy he developed erectile dysfunction which was well responding to Alprostradil 20 micrograms. Two years postoperatively the patient was admitted to A\&E with 8-week history of persistent and painful erection. On clinical examination the penis was tender and some nodules and warts were noted along the penile shaft and glans. The penile and pelvic MRI scan showed that both corpora cavernosa had severe fibrosis with thickening of the tunica and urethra throughout. The glans appeared abnormal with a number of exophitic lesions arising from it. Numerous bony metastases were also seen within the inferior pubic ramus and pubic symphysis and in the right acetabulum. A recurrent disease was noted at the level of the vescicourethral anastomosis. A computed tomography (CT) total body scan confirmed several small metastatic nodules within the lungs. The bone scan showed osteoblastic metastases in the lumbar spine and pelvis. In order to confirm the diagnosis a glans wedge biopsy and multiple trucut biopsies of both corpora were performed. The final histopathology report showed extensive infiltration by metastatic prostatic adenocarcinoma composed mainly of fused acinar structures (equivalent to Gleason 4 pattern) with focal lymphovascular and perineural invasion. After multidisciplinary team meeting discussion, he was offered a penile palliative radiotherapy and a pudendal nerve block to alleviate his pain and hormonal therapy.

Case 2: a 48 year old patient underwent radical cystoprostatectomy with extended lymph node dissection and formation of an ileal conduit for urothelial bladder carcinoma (pT4a pN0). A month later the patient was readmitted with 7 days history of ischemic priapism, refractory to primary treatment and $\mathrm{T}$ shunt. During the $\mathrm{T}$ shunt bilateral corporal biopsies were obtained and confirmed the presence of recurrence of primary disease. Following the multi disciplinary team meeting outcome the patient was offered a palliative penectomy followed by adjuvant chemotherapy. A palliative radical penectomy with formation of perineal urethrostomy was performed. The final histological report showed extensive infiltration along the entire length of the penis, the fea- 
tures are consistent with high grade urothelial carcinoma, with focal sarcomatoid differentiation.

Case 3: A 72 year old man, with a history of urothelial cell carcinoma of the bladder and concurrent incidental primary rectal adenocarcinoma in April 2008, underwent laparoscopic anterior resection and radical cystectomy with formation of ileal conduit. The bowel histology showed upper rectal G2pTl N1 disease fully resected- with no vascular invasion and the bladder histology showed G3pT4a (prostate) N2 disease with extensive vascular invasion. Therefore the patient had adjuvant chemotherapy. A CT scan and positron emission tomography (PET) post chemotherapy underlined a new lesion at right lower lobe of the lung. The biopsy of this lesion showed metastatic urothelial cancer. One year postoperatively the patient was admitted with a 4 day history of pain in his genitalia. The clinical examination revealed a semi-erect firm glans, with palpable tumour nodules. Abdomen MRI confirmed the presence of extensive pelvic lymph nodes. The clinical progression of priapism, with excruciating pain non responding to conventional medical therapy and conservative treatment, led to the medical decision for a palliative radical penectomy without any previous corporal biopsies, as the diagnosis of metastatic cancer was clear on the MRI scan.

\section{Discussion}

To date there are 512 published cases of secondary penile metastasis, as a result of the 118 founded in our Pubmed review of 64 published case reports of secondary penile metastasis in the last 10 years (from January 2004 to May 2014) searching for "penile metastasis", "penile metastases", "malignant priapism", "secondary malignancy penis", plus the 394 cases reviewed by Yu-Hsiang L. et al. In our review $157(30.6 \%)$ patients present metastasis of bladder origin, $152(29.6 \%)$ from prostate, $72(14 \%)$ recto-sigmoid, 34(6.6\%) kidney, 20(3.9\%) lower GI (excluding rectosigmoid), $15(2.9 \%)$ testis, $13(2.5 \%)$ lung, $11(2.1 \%)$ upper GI tract, 6(1.1\%) bone, 5(0.9\%) lymphoma, 4(0.7\%) leukemia, 4(0.7\%) melanoma, 4(0.7\%) upper airways, $3(0.5 \%)$ hepatobiliary, $2(0.3 \%)$ ureter, $1(0.1 \%)$ tongue, $1(0.1 \%)$ thyroid, $1(0.1 \%)$ seminal vesicle, $1(0.1 \%)$ glomangiosarcoma and $6(1.1 \%)$ from others origin. The most common clinical signs of penile metastases are, priapism $(40 \%)$, painful or painless penile nodules $(27.5 \%)$, swelling $(15 \%)$ pain $(7.5 \%)$, haematuria $(5 \%)$ and urinary retention (5\%) (1). The most common ways in which the primary cancer can spread to the penis are; the retrograde venous route due to the vast communication between the pelvic organs and dorsal venous system of the penis; via retrograde lymphatic flow and direct invasion from the close organs with advanced cancer. Direct arterial spread is another route, but less common. In our specific case the ischaemic priapism was due to the invasion of malignant cells into the cavernous sinuses blocking the venous draining veins, without blocking the arterial flow (2). According to Kendi et al., the MRI scan plays an important role in the diagnosis and stadiation (3). However the key point in order to confirm the diagnosis and the primary site of neoplasm is the biopsy of the

Table 1.

Primary site of penile metastases.

\begin{tabular}{|lcc|}
\hline Primary site & Number of cases & $\%$ \\
\hline Bladder & 157 & 30.6 \\
\hline Prostate & 152 & 29.6 \\
\hline Recto-sigmoid & 72 & 14 \\
\hline Kidney & 34 & 6.6 \\
\hline Lower GI tract (excluding recto-sigmoid) & 20 & 3.9 \\
\hline Testis & 15 & 2.9 \\
\hline Lung & 13 & 2.5 \\
\hline Upper Gl tract & 11 & 2.1 \\
\hline Bone & 6 & 1.1 \\
\hline Lymphoma & 5 & 0.9 \\
\hline Leukemia & 4 & 0.7 \\
\hline Melanoma & 4 & 0.7 \\
\hline Upper airways & 4 & 0.7 \\
\hline Hepatobiliary & 3 & 0.5 \\
\hline Ureter & 2 & 0.3 \\
\hline Thyroid & 1 & 0.1 \\
\hline Tongue & 1 & 0.1 \\
\hline Seminal vesicle & 1 & 0.1 \\
\hline Glomangiosarcoma & 1 & 0.1 \\
\hline Other & 612 & 1.1 \\
\hline Total & & \\
\hline
\end{tabular}

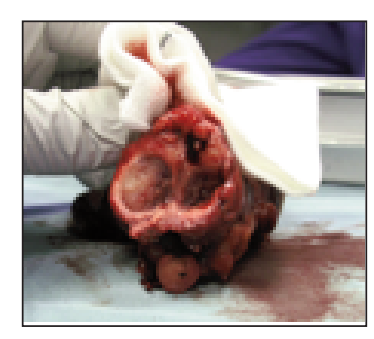

Figure 1.

Corpora cavernosa infiltrated by tumor.

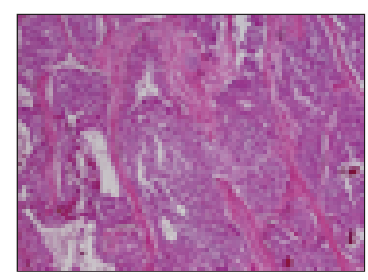

Figure 2.

Extensive permeation of the vascular channels of the corpus spongiosum and corpus cavernosum by TCC of the bladder.

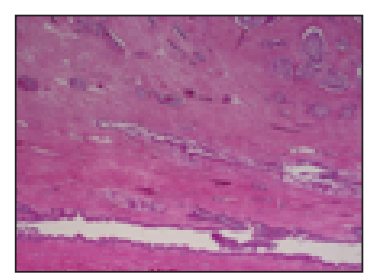

Figure 3.

Evidence of intravascular tumour thrombi.

corpora. Penile metastasis is an advanced stage related to a poor prognosis; the treatment is just palliative in order to improve the quality of life and reveal the pain. It could be hormone therapy, chemotherapy, radiotherapy or surgery (shunt or penectomy). The shunt surgery has limited success, especially if the duration of the iscahemic priapism is more than 48 hours. MDT(multidisciplinary team meeting) discussion should always be made for the treatment management in each case. The overall survival is 12 months (range 6-18 months) from the time of the diagnosis. 


\section{Conclusion}

Malignant priapism is a rare event. The most frequent primary sites are bladder, prostate and recto-sigmoid. Corporal biopsies are considered an effective method of diagnosis of the primary organ site.

\section{REFERENCES}

1. Lin YH, Kim JJ, Stein NB, Khera M. Malignant priapism second- ary to metastatic prostate cancer: a case report and review of literature. Rev Urol. 2011; 13:90-4.

2. Kumar N, Bhattacharyya T, Mandal AK, et al. Penile metastasis secondary to bladder cancer: a report of two cases. Indian J Palliat Care. 2014; 20:57-60.

3. Kendi T, Batislam E, Basar MM, et al. Magnetic resonance imaging (MRI) in penile metastases of extragenitourinary cancers. Int Urol Nephrol. 2006; 38:105-9.

\section{Correspondence}

Francesco De Luca, MD (Corresponding Author)

francescodelucal0@gmail.com

David Ralph, MD

dralph@andrology.co.uk

St Peter's Andrology and the Insitute of Urology

University College London Hospital, London, UK

Evangelos Zacharakis, MD

evangelos.zacharakis@doctors.org.uk

Department of Urology, Guy's Hospital

Kings College London, UK

Majed Shabbir, MD

majedshabbir@hotmail.com

Department of Urology, Guy's Hospital

Kings College London, UK

Angela Maurizi, MD

angmau81@hotmail.com

Carlo De Dominicis, MD

carlo.dedominicis@uniromal.it

Department of Gynecologic-Obstetric Sciences and Urological Sciences

Sapienza University of Rome, Rome, Italy

Emy Manzi, MD

emymanzi@hotmail.it

Department of General Surgery and Organ Transplantation

Sapienza University of Rome, Rome, Italy

Antonio Zanghì, MD

amzanghi@unict.it

General Surgery and Breast Unit, Department of Surgery

University of Catania, Catania, Italy 